\title{
Simple Derivation of Quasinormal Modes for Arbitrary Spins
}

Iosif KHRIPLOVICH and Gennady RUBAN

Budker Institute of Nuclear Physics, 630090 Novosibirsk, Russia

E-mail: khriplovich@inp.nsk.su,gennady-ru@ngs.ru

Received October 07, 2005, in final form November 05, 2005; Published online November 07, 2005

Original article is available at http://www.emis.de/journals/SIGMA/2005/Paper013/

\begin{abstract}
The asymptotically leading term of quasinormal modes (QNMs) in the Schwarzschild background, $\omega_{n}=-i n / 2$, is obtained in two straightforward analytical ways for arbitrary spins.
\end{abstract}

Key words: Regge-Wheeler equation; quasinormal modes

2000 Mathematics Subject Classification: 83C05; 83C45; 83C57

\section{Introduction}

The investigation of perturbations of various fields in the Schwarzschild background was started in $[1,2]$. Quasinormal modes (QNM) are the eigenmodes of the homogeneous wave equations, describing these perturbations, with the boundary conditions corresponding to outgoing waves at the spatial infinity and incoming waves at the horizon. The interest to QNMs was initiated by $[3,4]$.

Two boundary conditions make the frequency spectrum $\omega_{n}$ of QNMs discrete. The asymptotic form of this spectrum for gravitational and scalar perturbations of the Schwarzschild background was found initially in $[5,6]$ by numerical methods:

$$
\omega_{n}=-\frac{i}{2}\left(n+\frac{1}{2}\right)+0.087424, \quad n \rightarrow \infty, \quad s=0,2 .
$$

Here and below the gravitational radius $r_{g}$ is put to unity; $s$ is the spin of the perturbation. This result up to now serves as a touch stone for investigations in the field.

A curious observation was made in [7]: the real constant in (1) can be presented as

$$
\operatorname{Re} \omega_{n}=\frac{\ln 3}{4 \pi}=T_{\mathrm{H}} \ln 3,
$$

where $T_{\mathrm{H}}$ is the Hawking temperature $\left(T_{\mathrm{H}}=1 /(8 \pi k M) \text { in the common units }\right)^{1}$. Then, expression (2) for the asymptotic of $\operatorname{Re} \omega_{\mathrm{n}}$ was derived in [8] by solving approximately the recursion relations used previously in the numerical calculations. In the next paper [9] formula (2) was derived analytically. Besides, in [8] the following result was obtained for the asymptotic of QNMs for spin 1:

$$
\omega_{n}=-\frac{i}{2} n, \quad \operatorname{Re} \omega_{n} \rightarrow 0, \quad n \rightarrow \infty, \quad s=1 .
$$

\footnotetext{
${ }^{1}$ It was also conjectured in [7] that the asymptotic value (2) for Re $\omega_{n}$ is of a crucial importance for the quantization of gravitational field, fixing the value of the so-called Barbero-Immirzi parameter. In spite of being very popular, this idea is not in fact dictated by any sound physical arguments; quite the contrary, it is in conflict with them [10].
} 
While the results (1), (3) for integer $s$ are firmly established now, it is not the case for spin $1 / 2$. Two different approaches ${ }^{2}$ used in [11] result in the interval two times smaller than those for integer spins, namely:

$$
\omega_{n}=-\frac{i}{4} n, \quad n \rightarrow \infty, \quad s=1 / 2 .
$$

On the other hand, numerical calculations in [12] result in spectrum

$$
\omega_{n}=-\frac{i}{2}\left(n+\frac{1}{2}\right), \quad \operatorname{Re} \omega_{n} \rightarrow 0, \quad n \rightarrow \infty, \quad s=1 / 2 .
$$

One of the motivations of our work was the resolution of this discrepancy; we confirm below the leading term in equation (5).

\section{Quasinormal modes in Regge-Wheeler formalism}

Our derivation is based on the Regge-Wheeler equation treated to the leading approximation in large frequency $\omega,|\omega| \gg 1$.

In general, this equation for the radial function $\Psi$ corresponding to the angular momentum $j$ of a field with integer $\operatorname{spin} s(s=0,1,2 ; j \geq s)$ is written usually as

$$
\frac{d^{2} \Psi}{d z^{2}}+\left\{\omega^{2}-\left(1-\frac{1}{r}\right)\left[\frac{j(j+1)}{r^{2}}+\frac{1-s^{2}}{r^{3}}\right]\right\} \Psi=0 .
$$

Its analogue for $s=1 / 2$ (again the angular momentum $j \geq s$ ), written for the standard representation of the Dirac $\gamma$-matrices and states of definite parity, is

$$
\frac{d^{2} \Psi}{d z^{2}}+\left\{\omega^{2}-\left(1-\frac{1}{r}\right) \frac{(j+1 / 2)^{2}}{r^{2}}+\frac{\kappa}{2 r^{3}}\left(1-\frac{1}{r}\right)^{1 / 2}-\frac{\kappa}{r^{2}}\left(1-\frac{1}{r}\right)^{3 / 2}\right\} \Psi=0
$$

here $\kappa= \pm(j+1 / 2)$, with the sign depending on the parity of the state considered (this sign is irrelevant for our problem). The presence of the terms with fractional powers of $r$ and $r-1$ in equation (7) is quite natural since wave equations for half-integer spins are written via tetrads which are roughly square roots of metric $^{3}$.

In both equations, (6) and (7), $r$ is treated as a function of the so-called "tortoise" coordinate $z$. They are related as follows: $z=r+\ln (r-1)$, so that $z \rightarrow \infty$ for $r \rightarrow \infty$, and $z \rightarrow-\infty$ for $r \rightarrow 1$. The boundary conditions for QNMs of (6) and (7) are

$$
\Psi(z) \sim e^{ \pm i \omega z}, \quad z \rightarrow \pm \infty .
$$

For our purpose, it is convenient to go over in both equations, (6) and (7), to the usual coordinate $r$ and to new radial function $u(r)$ related to $\Psi$ as follows:

$$
\Psi=\frac{r^{1 / 2}}{(r-1)^{1 / 2}} u(r) .
$$

\footnotetext{
${ }^{2}$ We believe that one of them, despite being rather popular, can be dismissed at once. It is based on the analysis of the location of the poles of the scattering amplitude which by itself causes no objections. However, following [13, 14, 15], the authors of [11] analyze the poles of the corresponding Born amplitude. Meanwhile, the Born approximation by itself implies that the amplitude of the scattered wave is small. Therefore, its poles cannot have any meaning. Any coincidence between their position and that of the poles of a true amplitude is an accident only.

${ }^{3}$ We mention here one more rather popular belief, namely, that equation (6) applies to half-integer $s$ as well. The explicit difference between (6) and (7) demonstrates that this idea is wrong.
} 
The obtained equations for $u(r)$ can be rewritten as

$$
\begin{aligned}
& \frac{d^{2} u}{d r^{2}}+\left\{\omega^{2}+\frac{1}{r-1}\left[2 \omega^{2}-\left(j+\frac{1}{2}\right)^{2}+s^{2}-\frac{1}{4}\right]+\frac{1}{(r-1)^{2}}\left(\omega^{2}+\frac{1}{4}\right)\right. \\
&\left.+\frac{1}{r}\left[\left(j+\frac{1}{2}\right)^{2}-s^{2}+\frac{1}{4}\right]+\frac{1}{r^{2}}\left(-s^{2}+\frac{1}{4}\right)\right\} u=0, \quad s=0,1,2 \\
& \frac{d^{2} u}{d r^{2}}+\left\{\omega^{2}+\frac{1}{r-1}\left[2 \omega^{2}-\left(j+\frac{1}{2}\right)^{2}+\frac{1}{2}\right]+\frac{1}{(r-1)^{2}}\left(\omega^{2}+\frac{1}{4}\right)\right. \\
&\left.\quad+\frac{1}{r}\left[\left(j+\frac{1}{2}\right)^{2}-\frac{1}{2}\right]-\frac{3}{4} \frac{1}{r^{2}}-\frac{\kappa}{r^{3 / 2}(r-1)^{1 / 2}}+\frac{1}{2} \frac{\kappa}{r^{3 / 2}(r-1)^{3 / 2}}\right\} u=0, \quad s=1 / 2
\end{aligned}
$$

We are interested in the solutions of equations (10) and (11) in the interval $1<r<\infty$ for $|\omega| \rightarrow \infty$. Obviously, all the terms singular at $r \rightarrow 0$, in both these equations, are relatively small in this interval if $|\omega| \rightarrow \infty^{4}$. Therefore, these terms can be safely omitted, and we arrive at the following universal truncated wave equation for all spins:

$$
\frac{d^{2} u}{d r^{2}}+\left[\omega^{2}+\frac{2 \omega^{2}}{r-1}+\frac{\omega^{2}+1 / 4}{(r-1)^{2}}\right] u=0
$$

We have omitted here also the terms $-(j+1 / 2)^{2}+s^{2}$ and $-(j+1 / 2)^{2}+1 / 2$ in the coefficients at $1 /(r-1)$ in (10) and (11), respectively. Though these terms could be easily included into the solutions, they would result in corrections to $\operatorname{Im} \omega_{n}$ on the order of $1 / n$ only, which are negligible as compared to the leading term $\sim n$.

We retain however the term $1 / 4$ in the coefficient at $1 /(r-1)^{2}$ in (12). Otherwise the wave function asymptotic for $z \rightarrow-\infty$ would be $e^{-i \omega z+1 / 2}$, instead of $e^{-i \omega z}$. In other words, the effective potential in the initial Regge-Wheeler equations (6), (7) would not vanish for $z \rightarrow-\infty$, but would tend instead to $1 / 4$. Indeed, the wave function asymptotic for $z \rightarrow-\infty$ is determined by the discussed coefficient at $1 /(r-1)^{2}$. Since the coefficient $\omega^{2}+1 / 4$ at $1 /(r-1)^{2}$ in (12) corresponds to $\omega^{2}$ in equations (6), (7), then obviously the coefficient $\omega^{2}$ in (12) would correspond to $\omega^{2}-1 / 4$ in $(6),(7)$.

To summarize, it is only natural that equation (12), essentially semiclassical one (due to the assumption $|\omega| \gg 1$ ), is universal, i.e. independent of spin $s$. Moreover, even if one assumes that $j \gg 1$ as well (i.e. gives up the condition $j \ll|\omega|$ used in (12)), the resulting, again semiclassical equation

$$
\frac{d^{2} u}{d r^{2}}+\left\{\omega^{2}+\frac{2 \omega^{2}}{r-1}+\frac{1}{(r-1)^{2}}\left(\omega^{2}+\frac{1}{4}\right)-\frac{1}{r(r-1)}\left(j+\frac{1}{2}\right)^{2}\right\} u=0
$$

is still universal, i.e. spin-independent.

We address now the eigenvalues of equation (12). Its two independent solutions can be conveniently expressed via the Whittaker functions $W_{\lambda, \mu}(x)[16]$. They are

$$
W_{i \omega, i \omega}(-2 i \omega(r-1)), \quad W_{-i \omega, i \omega}(2 i \omega(r-1)) .
$$

\footnotetext{
${ }^{4}$ In particular, in equation (11)

$$
|\kappa| r^{-3 / 2}(r-1)^{-1 / 2} \ll\left|\omega^{2}\right|(r-1)^{-1}, \quad \text { and } \quad|\kappa| r^{-3 / 2}(r-1)^{-3 / 2} \ll\left|\omega^{2}\right|(r-1)^{-2}
$$
}

for the interval $1<r<\infty$. 


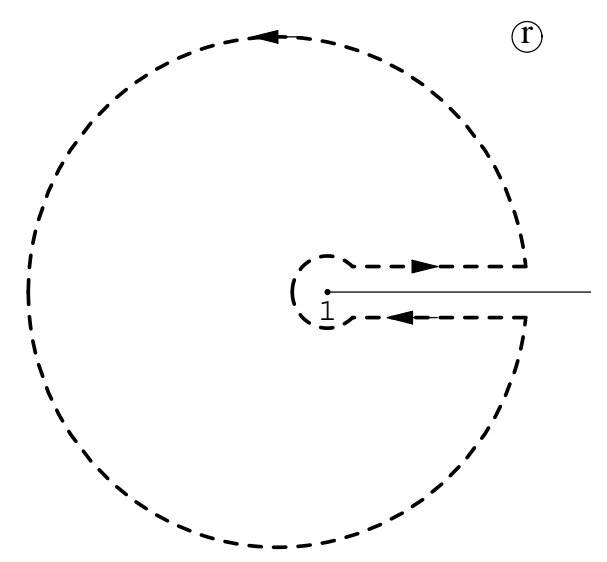

Figure 1. Closed contour in the complex $r$ plane.

With their different asymptotic for $r \rightarrow \infty$,

$$
\begin{aligned}
& W_{i \omega, i \omega}(-2 i \omega(r-1)) \rightarrow e^{i \omega[r+\ln (r-1)]}=e^{i \omega z}, \\
& W_{-i \omega, i \omega}(2 i \omega(r-1)) \rightarrow e^{-i \omega[r+\ln (r-1)]}=e^{-i \omega z},
\end{aligned}
$$

these solutions are obviously independent. On the other hand, the second one does not comply with boundary condition (8) and therefore should be excluded.

As to the first solution, its limit for $r \rightarrow 1$ is

$$
\begin{aligned}
& W_{i \omega, i \omega}(-2 i \omega(r-1)) \longrightarrow \\
& \quad \longrightarrow \frac{\Gamma(-2 i \omega)}{\Gamma(1 / 2-2 i \omega)}[-2 i \omega(r-1)]^{i \omega+1 / 2}+\frac{\Gamma(2 i \omega)}{\Gamma(1 / 2)}[-2 i \omega(r-1)]^{-i \omega+1 / 2} .
\end{aligned}
$$

When going over to the function $\Psi$ used in the "tortoise" coordinate $z$ (see (9)), the overall factor $(r-1)^{1 / 2}$ in this expression cancels, and $(r-1)^{ \pm i \omega}$ goes over into $e^{ \pm i \omega z}$ for $r \rightarrow 1$. To comply with the boundary condition on the horizon, one should get rid of the first term in equation (14). To this end, recalling that $\Gamma(-n)$ has poles for integer positive $n$, we put $1 / 2-2 i \omega=-n$, or $\omega_{n}=-(i / 2)(n+1 / 2)$.

In fact, equation (12) by itself was obtained from (10) and (11) under the assumption $\left|\omega_{n}\right| \rightarrow \infty$, or $n \gg 1$. Therefore, in this way we can guarantee, for the initial problem, only that

$$
\omega_{n}=-\frac{i}{2} n, \quad n \gg 1
$$

for all spins. Though less accurate than quantization rules (1), (3), and (5), this one is still quite sufficient for insisting that the correct quantization rule for spin $1 / 2$ is (5), but not (4).

\section{Alternative derivation of quasinormal modes}

The same result can be obtained otherwise, without resorting to special functions. This derivation is as follows.

Equation (12) has two singular points, $r=1$ and $r=\infty$. We connect them by a cut in the complex plane $r$ going, for instance, from $r=1$ along the real axis to the right (see Fig. 1). Let us consider the closed contour marked by the dashed line in Fig. 1. Since there is no singularity inside it, the solution at some point on this contour, after going around the contour, comes back 
to its initial value, which means that the phase of this solution changes by $2 \pi n, n=0, \pm 1, \pm 2, \ldots$. When we follow an arc of a large radius $r \gg 1$, where the asymptotic solution is $e^{i \omega r} r^{i \omega}$, i.e. go around the singular point at infinity, the wave function acquires the phase $\delta(\infty)=2 \pi i \omega$. Then we go around the branch point $r=1$ by following an arc of a small radius. Here the asymptotic solution is $(r-1)^{-i \omega+1 / 2}$, and the wave function acquires the phase $\delta(1)=2 \pi(i \omega-1 / 2)$. As to the paths along the cut, no phase at all is acquired along them. Indeed, this cut is due to the mentioned asymptotic solution $v(r)=(r-1)^{-i \omega+1 / 2}$, and therefore the wave function can be written as $u(r)=v(r) w(r)$, where $w(r)$ is analytic at $r=1$, i.e. has no cut at all. The phase acquired by $u(r)$ is obviously the sum of phases acquired by $v(r)$ and $w(r)$. However, along the paths adjacent to the cut, the phase of $v(r)=(r-1)^{-i \omega+1 / 2}$ remains constant, as well as that of $r-1$. As to the analytic function $w(r)$, along the path from 1 to $\infty$ its phase can vary, but this change will be cancelled exactly by that acquired when going in the opposite direction, from $\infty$ to 1 . In other words, effectively for our purpose, the branch point $r=1$ behaves as if it were an isolated singularity.

Thus, going counter-clockwise around the considered closed contour in the complex plane, one obtains

$$
\delta(\infty)+\delta(1)=4 \pi i \omega-\pi=2 \pi n,
$$

or the quantization rule

$$
\omega_{n}=-\frac{i}{2}(n+1 / 2) .
$$

Being interested in the solutions decreasing in time, we choose here positive $n$ (and of course large ones).

\section{Acknowledgements}

We are grateful to V.M. Khatsymovsky, A.A. Pomeransky, and V.V. Sokolov for the interest to this work and useful discussions. The investigation was supported in part by the Russian Foundation for Basic Research through Grant No. 05-02-16627.

[1] Regge T., Wheeler J.A., Stability of a Schwarzschild singularity, Phys. Rev., 1957, V.108, $1063-1069$.

[2] Zerilli F.J., Gravitational field of a particle falling in a Schwarzschild geometry analyzed in tensor harmonics, Phys. Rev. D, 1970, V.2, 2141-2160.

[3] Vishveshwara C.V., Scattering of gravitational radiation by a Schwarzschild black hole, Nature, 1970, V.227, 936-938.

[4] Press W.H., Long wave trains of gravitational waves from a vibrating black hole, Astrophys. J., 1971, V.170, L105.

[5] Leaver E.W., An analytic representation for the quasi-normal modes of Kerr black holes, Proc. Roy. Soc. London Ser. A, 1985, V.402, 285-298.

[6] Nollert H.-P., Quasinormal modes of Schwarzschild black holes: the determination of quasinormal frequencies with very large imaginary parts, Phys. Rev. D, 1993, V.47, 5253-5258.

[7] Hod S., Bohr's correspondence principle and the area spectrum of quantum black holes, Phys. Rev. Lett., 1998, V.81, 4293-4296; gr-qc/98120072.

[8] Motl L., An analytical computation of asymptotic Schwarzschild quasinormal frequencies, Adv. Theor. Math. Phys., 2002, V.6, 1135-1162; gr-qc/0212096.

[9] Motl L., Neitzke A., Asymptotic black hole quasinormal frequencies, Adv. Theor. Math. Phys., 2003, V.7, 307-330; hep-th/0301173.

[10] Khriplovich I.B., Quasinormal modes, quantized black holes, and correspondence principle, Int. J. Mod. Phys. D, 2005, V.14, 181-183; gr-qc/0407111.

[11] Castello-Branco K.H.C., Konoplya R.A., Zhidenko A., High overtones of Dirac perturbations of a Schwarzschild black hole, Phys. Rev. D, 2005, V.71, 047502, 4 pages; hep-th/0411055. 
[12] Jing J., Dirac quasinormal modes of Schwarzschild black hole, Phys. Rev. D, 2005, V.71, 124006,7 pages; gr-qc/0502023.

[13] Padmanabhan T., Quasi normal modes: a simple derivation of the level spacing of the frequencies, Class. Quant. Grav., 2004, V.21, L1-L6; gr-qc/0310027.

[14] Medved A.J.M., Martin D., Visser M., Dirty black holes: quasinormal modes, Class. Quant. Grav., 2004, V.21, 1393-1405; gr-qc/0310009.

[15] Roy Chaudhury T., Padmanabhan T., Quasinormal modes in Schwarzschild-de Sitter spacetime: a simple derivation of the level spacing of the frequencies, Phys. Rev. D, 2004, V.69, 064033, 9 pages; gr-qc/0311064.

[16] Gradshteyn I.S., Ryzhik I.M., Table of integrals, series and products, New York, Academic Press, 1994. 